\title{
Rap1A promotes esophageal squamous cell carcinoma metastasis through the AKT signaling pathway
}

\author{
QINFANG LI $^{1 *}$, AIPING XU $^{1 *}$, YUAN CHU $^{1}$, TAO CHEN $^{1}$, HONGQI LI $^{1}$, \\ LIQING YAO ${ }^{1}$, PINGHONG ZHOU ${ }^{1}$ and MEIDONG XU ${ }^{1,2}$ \\ ${ }^{1}$ Endoscopy Center and Endoscopy Research Institute, Zhongshan Hospital, Fudan University, Shanghai 200032; \\ ${ }^{2}$ Endoscopy Center, Shanghai East Hospital, Tongji University School of Medicine, Shanghai 200120, P.R. China
}

Received December 16, 2018; Accepted August 16, 2019

DOI: $10.3892 /$ or.2019.7309

\begin{abstract}
Ras-associated protein 1A (Rap1A) is a member of the Ras subfamily of small GTP-binding proteins and is found to promote metastasis in several types of cancer. However, the functional role and molecular mechanism of action in Rap1A in esophageal squamous cell carcinoma (ESCC) is not fully understood. In the present study, Rap1A was found to be upregulated in ESCC tissues and its expression was correlated with cancer stage. Functional studies revealed that Rap1A could promote ESCC metastasis by stimulating cell migration and invasion in vivo and in vitro. Further study indicated that the transcriptional factor SP1 increased Rap1A expression via promoter binding and transcription activation. Furthermore, Rap1A promoted epithelial-to-mesenchymal transition, possibly through the AKT signaling pathway. Hence, the findings of the present study indicated that Rap1A may be a potential prognostic marker or therapeutic target for ESCC.
\end{abstract}

\section{Introduction}

Esophageal squamous cell carcinoma (ESCC) is a malignancy that most commonly presents with progressive dysphagia. It has been reported to be the fourth leading cause of cancer-related mortality and the third most common type of cancer in China, accounting for over half of all esophageal cancer cases worldwide (1). When diagnosed at an early stage, when dysplasia is limited to the mucosal layer and there is no lymph node metastasis, ESCC may be curable by endoscopic resection (2); however, since the majority of patients with early-stage disease are asymptomatic, ESCC is often diagnosed at an advanced

Correspondence to: Dr Meidong Xu, Endoscopy Center and Endoscopy Research Institute, Zhongshan Hospital, Fudan University, 180 FengLin Road, Shanghai 200032, P.R. China

E-mail: zsxumeidong@yahoo.com

*Contributed equally

Key words: Ras-associated protein 1A, AKT, esophageal squamous cell carcinoma, metastasis stage, when it is most likely metastatic, and the overall 5-year survival rate ranges from 15 to $25 \%(3,4)$. Therefore, identification of the mechanism underlying esophageal cancer metastasis is crucial for treatment selection.

The phosphoinositide 3-kinase (PI3K)/AKT/mammalian target of rapamycin (mTOR) signaling pathway is one of the most frequently activated pathways in human cancers, including ESCC (5-9). In mammals, PI3Ks may be classified into class I, II and III, according to their structure and substrate specificity. Class I PI3Ks consist of a regulatory subunit (p85, p101 or p84) and a catalytic subunit (p110 $\alpha$, $\mathrm{p} 110 \beta, \mathrm{p} 110 \gamma$ or $\mathrm{p} 1108$ ). GTP-Ras interacts with $\mathrm{p} 85$ to induce a p110 conformational change to phosphorylate PI3K. PIP3, the product of class I PI3K, recruits the serine-threonine kinase AKT, which contains a pleckstrin homology $(\mathrm{PH})$ domain, and its activating kinase 3-phosphoinositide-dependent kinase 1 (PDK1) to cell membranes, allowing PDK1 to activate AKT (10). AKT signals regulate multiple oncogenic processes, including cell proliferation, differentiation, apoptosis, epithelial-to-mesenchymal transition (EMT), migration and invasion (11).

Ras-associated protein 1A ( Rap1A) is a type of small GTP-binding protein that belongs to the Ras subfamily. Similar to other Ras proteins, Rap1A switches between an inactive GDP-bound and an active GTP-bound form. Although Ras and Rap1A share almost the same effectors near the cell surface, Rap1A is activated in the perinuclear region, while the majority of Ras proteins are activated at the plasma membrane $(12,13)$. Previous research has demonstrated that Rap1A plays an essential regulatory role in a variety of cellular processes, including proliferation, integrin activation, cell adhesion and migration. However, the specific function of Rap1A varies by cell type; for example, Rap1A mediates integrin-dependent glioblastoma cell proliferation and tumor growth (14). Rap1A also plays an important role in prostate cancer cell migration and invasion $(15,16)$ and promotes ovarian cancer cell proliferation, migration and invasion (17). These results suggest that Rap1A may act as an oncogene; however, the specific role and mechanism of action of Rap1 A in ESCC remains unknown. In the present study, Rap1 A was silenced in ECA109 and KYSE150 ESCC cells and its functions were examined in vivo and in vitro. The underlying molecular mechanism was also investigated. 


\section{Materials and methods}

Cell lines and culture conditions. The human esophageal squamous cancer cell lines ECA109 and KYSE150 were purchased from Genechem Co., Ltd. All cells were maintained at $37^{\circ} \mathrm{C}$ with $5 \% \mathrm{CO}_{2}$. ECA109 cells were grown in DMEM (SH30243.01; HyClone; GE Healthcare Life Sciences) and KYSE150 cells were maintained in RPMI-1640 medium (SH30809.01; HyClone; GE Healthcare Life Sciences), both of which were supplemented with $10 \%$ fetal bovine serum (FBS; 10270106; Gibco; Thermo Fisher Scientific, Inc.) and penicillin-streptomycin solution.

Clinical samples and immunohistochemistry (IHC). A total of 170 paraffin-embedded ESCC specimens and adjacent non-tumor tissues (ANTT) acquired between 2006 and 2008 were obtained from the Fudan University-Affiliated Zhongshan Hospital. The inclusion criteria were as follows: i) No radiotherapy, chemotherapy or targeted therapy prior to surgery; ii) the results of pathological examination were in accordance with the clinical diagnosis; iii) complete clinicopathological data were available. The TNM classification was performed according to the eighth edition staging system published by the Journal of Thoracic Oncology (18). The details of 95 patients who provided ESCC samples are listed in supplementary Tables SI and SII. Rap1A antibody (rabbit polyclonal, 1:100 dilution; ab197673; Abcam) for IHC detection. Scoring was determined by two independent pathologists by combining the proportion of positively stained tumor or epithelial cells and the intensity of staining. The positively stained cell proportions were scored as follows: $0,<1 \%$ positive cells; $1,1-25 \%$ positive cells; $2,25-50 \%$ positive cells; $3,50-75 \%$ positive cells; and $4,>75 \%$ positive cells. Staining intensity was graded as follows: 0 , no staining; 1 , weak staining (light yellow); 2 , moderate staining (yellowish brown); 3 , strong staining (brown). The staining index (SI) was calculated as the product of the staining intensity score and the proportion of positive cells. Samples with possible SI scores of $0,1-4,5-8$ or 9-12 were considered to have negative, low, intermediate and strong expression, respectively.

In addition, IHC staining for Rap1A was performed on mouse lung sections to verify whether pulmonary nodules were formed by metastasis of ECA109 cells injected into the nude mice through the tail vein. The detailed procedures of IHC staining were as follows: Lung sections $(4-\mu \mathrm{m})$ were deparaffinized in xylene and rehydrated through an alcohol gradient; endogenous peroxidase was inactivated by hydrogen peroxide; antigen was retrieved by ethylenediaminetetraacetic acid (EDTA); tissue was blocked by goat serum for $30 \mathrm{~min}$, incubated with Rap1A antibody overnight at $4^{\circ} \mathrm{C}$ and finally incubated with secondary antibody for $20 \mathrm{~min}$ at room temperature; IHC reaction was visualized by DAB solution and the cell nuclei were stained by hematoxylin; the sections were dehydrated, transparentized and sealed for further examination or archiving.

Plasmid and lentivirus construction and cell transfection. To knock down the expression of Rap1A in ECA109 and KYSE150 cells, four shRNAs targeting human Rap1A or SP1 were designed, synthesized and inserted into the construct
U6-Rap1A-shRNA1/2/3/4-Ubi-Cherry-vector by Genechem. Cells were transfected with the plasmids described above using Lipofectamine 2000 (11668-019, Invitrogen; Thermo Fisher Scientific, Inc.) according to the manufacturer's protocol. For each Rap1A shRNA candidate, mRNA and protein expression was analyzed and the siRNAs targeting the sequences AAAGTCAAAGATCAATGTT and CAGCAT TCCAGACTTCAAA were determined as the two most effective for inhibiting Rap1A expression. Lentiviruses harboring the corresponding shRNAs were generated by transfection of 293T cells with a three-plasmid-based lentiviral packaging system. Stable ECA109 and KYSE150 cell lines with Rap1A silencing were generated via lentivirus infection and used for subsequent experiments. Moreover, the SP1 coding sequence was subcloned into the GV492 vector (Genechem) to construct a cDNA-SP1 plasmid and investigate its role in Rap1 A expression.

Reverse transcription-quantitative polymerase chain reaction $(R T-q P C R)$ analysis. Total cellular RNA was extracted using TRIzol reagent (15596-026; Ambion; Thermo Fisher Scientific, Inc.) and was reverse-transcribed to cDNA using the PrimeScript ${ }^{\mathrm{TM}}$ RT Master Mix (RR036A; Takara) according to the manufacturer's instructions. Subsequently, two-step RT-qPCR using TB Green (RR420A, Takara) was performed to determine mRNA levels. PCR was run using the following protocol: Initial activation at $95^{\circ} \mathrm{C}$ for $30 \mathrm{sec}$, followed by 40 cycles at $95^{\circ} \mathrm{C}$ for $5 \mathrm{sec}$ and at $60^{\circ} \mathrm{C}$ for $30 \mathrm{sec}$. The PCR primers used were as follows: Rap1A forward, $5^{\prime}$-TGTCTC ACTGCACCTTCA-3' and reverse, 5'-GACTTCCCAACG CCTCCT-3'; SP1 forward, 5'-TGGCAGCAGTACCAATGG C-3' and reverse, 5'-CCAGGTAGTCCTGTCAGAACTT-3'. All reactions were performed in triplicate and the mRNA expression was analyzed using the $2^{-\Delta \Delta \mathrm{Cq}}$ method (16).

Growth curves and colony formation assay. For the growth curves, cells were detached from flasks using $0.25 \%$ Trypsin-EDTA and centrifuged at $80 \mathrm{xg}$, room temperature for 5 min, counted, seeded at 3,000 cells per well (in quintuplicate) in 96-well culture plates in $100 \mu \mathrm{l}$ of recommended medium and cultured for 1, 2, 3, 4, 5 and 6 days. Cell growth was detected using MTT solution and DMSO, according to the manufacturer's instructions (ST316; Beyotime Institute of Biotechnology). The OD values at $490 \mathrm{~nm}$ were recorded and analyzed to compare the proliferation among cells. For the colony formation assay, cells were seeded $(300$ cells/well in triplicate) in 6-well plates and cultured for 2-3 weeks. Fresh culture medium was replaced every 3 days. When the colonies were detectable macroscopically, the cells were fixed and stained using $0.1 \%$ crystal violet solution (C0121; Beyotime Institute of Biotechnology). The colony-forming ability was evaluated using the colony number.

Transwell migration and invasion assay. To evaluate cell migration and invasion ability, 24-well Transwell insert chambers with $8.0-\mu \mathrm{m}$ polycarbonate membranes (3422; Corning, Inc.) were used. For migration assays, $5 \times 10^{4}$ cells were plated into the upper chamber in serum-free medium, and medium supplemented with $10 \%$ FBS was added to the lower chamber. Following incubation for $24 \mathrm{~h}$, the cells were fixed in ethanol 
for $0.1 \%$ crystal violet staining (C0121; Beyotime Institute of Biotechnology). The cells in the upper chamber were then removed using a cotton swab. Cell migration ability was analyzed by counting the number of stained cells under a light microscope. For invasion assays, after starvation for $24 \mathrm{~h}$, $1 \times 10^{5}$ cells were seeded in serum-free medium into the upper chamber that was precoated with Matrigel (354480; Corning, Inc.), and medium supplemented with $10 \%$ FBS was added to the lower chamber to serve as a chemoattractant. Following incubation for $48 \mathrm{~h}$, the cells were fixed, stained (non-invading cells were removed with a cotton swab), and analyzed as described above.

Western blotting. The cells were lysed using RIPA lysis buffer (P0013B; Beyotime Institute of Biotechnology), containing a protease and phosphatase inhibitor cocktail. Lysate protein concentrations were determined using a BCA kit (P0010S; Beyotime Institute of Biotechnology). The denatured protein samples were separated by $10 \%$ SDS-PAGE in electrophoresis buffer and transferred to a PVDF membrane. The membranes were blocked in 5\% milk for $2 \mathrm{~h}$, then incubated with primary antibodies overnight at $4^{\circ} \mathrm{C}$. The antibodies were directed against Rap1A (rabbit polyclonal, ab197673, 1:1,000; Abcam), $\beta 1$ integrin (rabbit polyclonal, ab183666, 1:3,000; Abcam), matrix metalloproteinase (MMP)9 (rabbit polyclonal, 10375-2-AP, 1:1,000; ProteinTech Group, Inc.), E-cadherin (rabbit monoclonal, 24E10, 1:1,000; Cell Signaling Technology, Inc.), Slug (rabbit polyclonal, arg55242, 1:1,000; Abcam), AKT (rabbit polyclonal, arg56418, 1:600; Arigo), SP1 (rabbit polyclonal, ab13370, 1:3,000) and pAKTser473 (rabbit monoclonal, 4060, 1:2,000; Cell Signaling Technology, Inc.). The membranes were incubated with HRP-conjugated secondary antibodies for $2 \mathrm{~h}$, and then antigen-antibody complexes were detected using an electrochemiluminescence kit to compare the corresponding protein expression levels among different samples.

Chromatin immunoprecipitation (ChIP) assay. All viable cells $\left(>2 \times 10^{6}\right)$ were treated with $1 \%$ formaldehyde to cross-link proteins to DNA. The DNA compounds were digested by a micrococcal nuclease (26156; Thermo Fisher Scientific, Inc.) into 100-1,000-bp segments. Equal aliquots of chromatin supernatants were incubated with anti-IgG antibody, anti-SP1 antibody (ab13370), or anti-RNA polymerase II antibody overnight at $4^{\circ} \mathrm{C}$ with rotation. Antibody-protein-DNA complexes were precipitated by protein A agarose and reverse-cross-linked to free the DNA. PCR and agarose gel electrophoresis were used to verify the protein-binding site on the DNA. The primers used were as follows: F1 forward, 5'-TTTCTGGCTGGGGTA AACCG-3' and reverse, 5'-GCTGGCACACTTTGTCTTGGA GC-3'; F2 forward, 5'-GGCTCAGGAGTCATAGCT-3' and reverse, 5'-GCATGTCCAAATTAACT-3'.

Luciferase reporter assay. Fragments containing the Rap1A promoter region or mutants of the predictive SP1-binding site were inserted upstream of the firefly luciferase coding sequences in the pGL3-basic reporter plasmid. ECA109 and KYSE150 cells were seeded onto 96-well plates (in triplicate), and were then transiently transfected with pGL3-ctrl, pGL3-Rap1A promoter, pGL3-Rap1A mut1, or pGL3-Rap1A mut2 and pTK-Renilla, with or without cDNA-SP1 plasmid. Fluc and Rluc activities were determined using the Dual-Glo Luciferase Assay System (E2920; Promega Corporation).

Tumor growth and metastasis in vivo. Female athymic nude mice (4-week-old, 16-18 g, n=12; and 5-week-old, 19-21 g, $\mathrm{n}=12$ ) were purchased from the Shanghai Laboratory Animal Center, Chinese Academy of Sciences. The mice were housed under specific pathogen-free conditions at the Fudan University Laboratory Animal Division. Briefly, for xenograft tumors, $5 \times 10^{6}$ cells (ECA109-shRap1A-NC or ECA109-shRap1A-4) were injected into the right dorsal flank of nude mice $(n=6$ per group). The length and width of the tumors were measured twice weekly using calipers and the tumor volume was calculated using the equation (length $\mathrm{x}$ width $\left.{ }^{2}\right) / 2$. When the length of any tumor reached $20 \mathrm{~mm}$ or greater, all animals were sacrificed and the tumors were excised. For lung metastasis, $1 \times 10^{6}$ cells were injected into the tail vein of nude mice $(n=6$ per group in two groups). The animals were sacrificed by cervical dislocation and the lungs were excised after 6 weeks or when the animals developed significant ascites or cachexia/emaciation, hind-limb paralysis or weakness. The nodules formed in the lungs were counted, and the lungs were then fixed in formalin and embedded in paraffin for further $\mathrm{H} \& \mathrm{E}$ and IHC staining.

The details of IHC staining were mentioned above, and $\mathrm{H} \& \mathrm{E}$ staining was performed as described below. After hydration, the sections were stained with hematoxylin for $10 \mathrm{~min}$. The cytoplasm was visualized by incubating with eosin for $\sim 2$ min. Finally, the sections were dehydrated, transparentized and sealed in the same manner as for IHC staining. In the present study, the subcutaneous and tail vein injections were performed without anesthesia. At the end of the animal experiment, cervical dislocation was used for euthanasia and death was verified by cardiac arrest.

Statistical analysis. All data were statistically analyzed using SPSS 20.0 (IBM Corp.) and GraphPad Prism 5.0 software (GraphPad Software, Inc.). For tumor volume, data are presented as the mean $+95 \%$ confidence interval $(\mathrm{CI})$, while the remaining data are shown as the mean \pm standard error of the mean. Statistical tests included the Student's t-test, $\chi^{2}$ test, ANOVA and Dunnett's post hoc test. $\mathrm{P}<0.05$ was considered to indicate statistically significant differences.

\section{Results}

RaplA expression is upregulated in ESCC. Rap1A is upregulated in cancer tissues compared with ANTT and contributes to the progression of prostate, colorectal and oral cavity squamous cell carcinoma (19-21). To investigate the expression and biological role of Rap1A in human ESCC, we examined the Rap1A expression pattern in 95 human ESCC and 75 ANTT samples. As shown in Fig. 1A and B, Rap1A was markedly overexpressed in human ESCC at the protein level $(\mathrm{P}<0.001)$. Furthermore, statistical analysis of the ESCC samples revealed a correlation between lymph node metastasis and Rap1A expression (Fig. 1C), i.e., Rap1A levels were markedly correlated with the N stage of human ESCC. Moreover, Rap1A expression was higher in N2 compared with N1 stage ESCC (Fig. 1D), suggesting that Rap1A may be a potential 

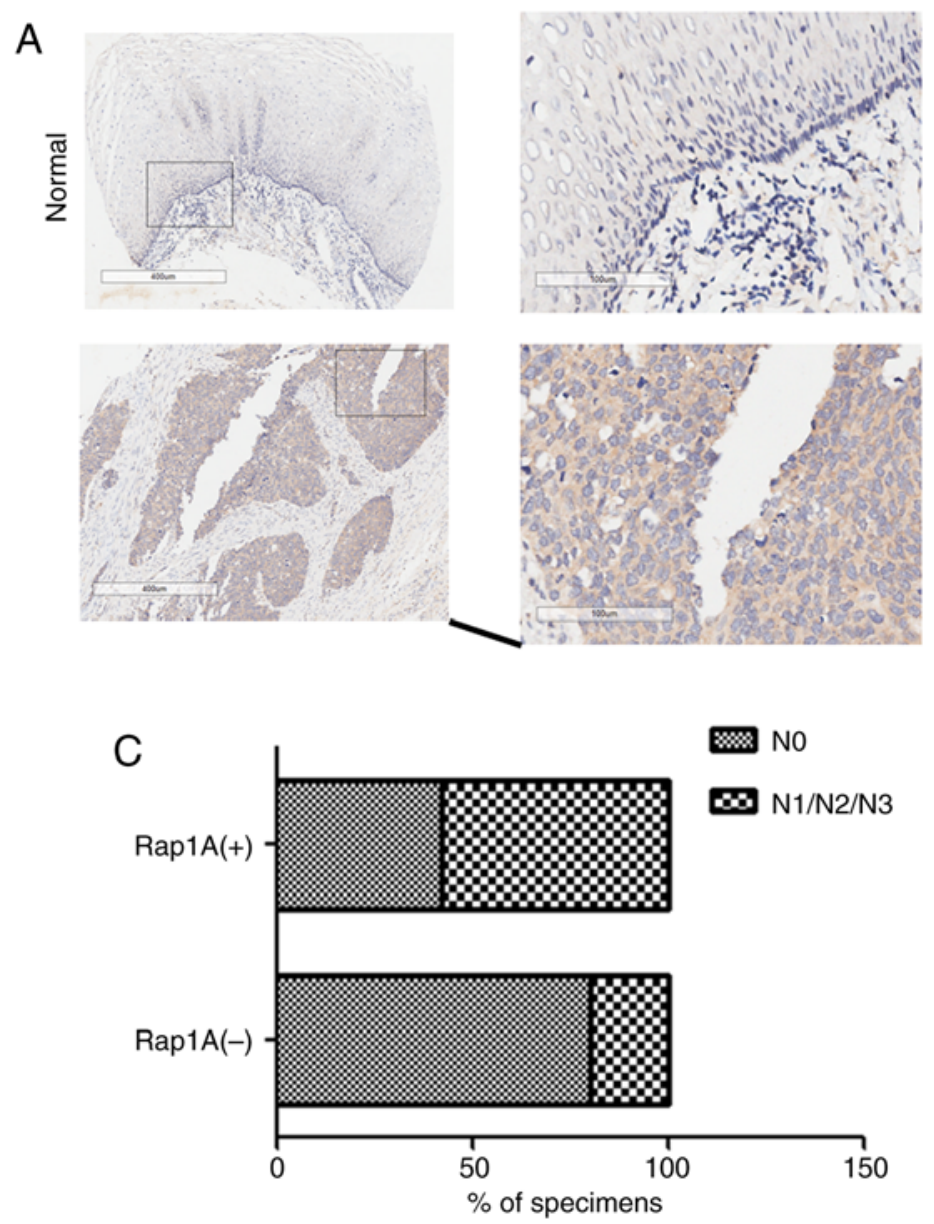
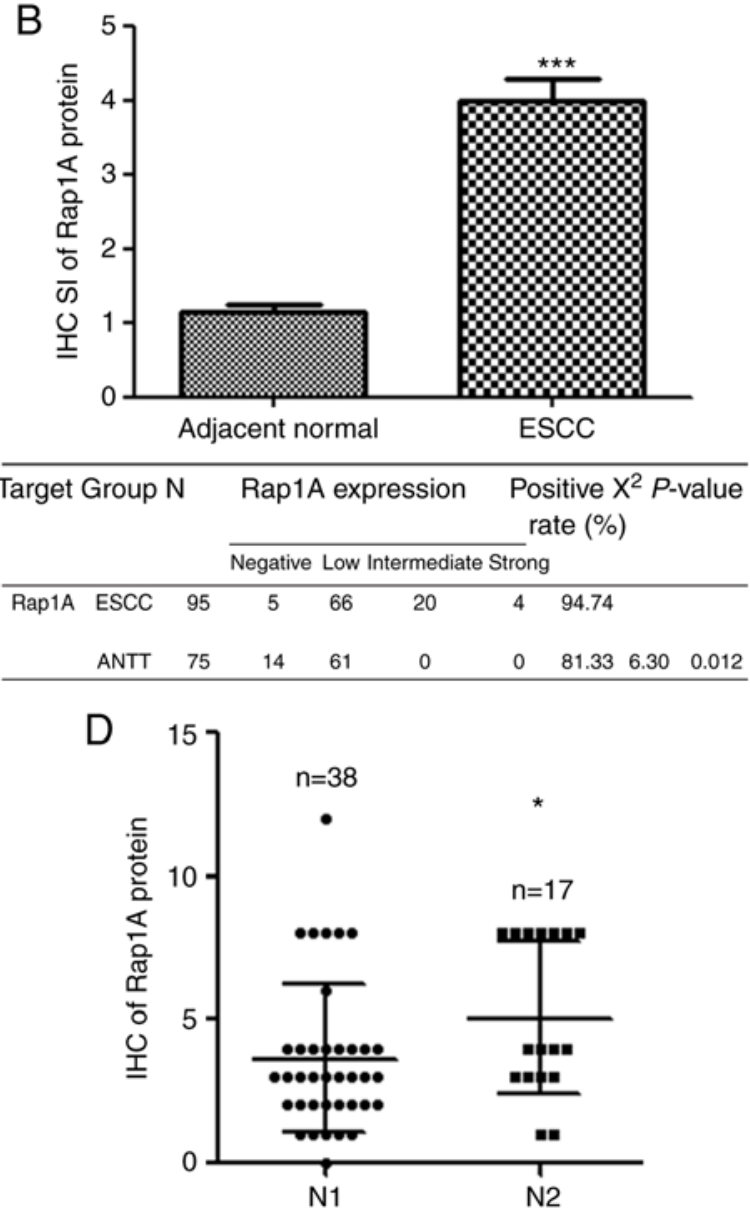

Figure 1. The expression of Rap1A is upregulated in ESCC. (A) IHC staining images. Scale bars, $400 \mu \mathrm{m}$; insets: $100 \mu \mathrm{m}$. (B) IHC analysis shows that the Rap1A expression is upregulated in human ESCC tissues compared with normal esophageal tissues. (C) Chi-squared test indicates statistically significant differences in the lymph node metastasis between the positive and negative Rap1A groups. (D) IHC analysis of the Rap1A expression in different lymph node stages of ESCC samples; error bars represent the mean \pm standard error of the mean; ${ }^{*} \mathrm{P}<0.05,{ }^{* * *} \mathrm{P}<0.001$. Rap1A, Ras-associated protein $1 \mathrm{~A}$; ESCC, esophageal squamous cell carcinoma; IHC, immunohistochemistry.

biomarker for the development and progression of lymph node metastasis in ESCC.

The growth-promoting effect of RaplA in ESCC is context-dependent. In order to elucidate the function of Rap1A in human ESCC, four types of shRNA targeting human Rap1A mRNA were screened using ECA109 cells (Fig. 2A and B) and the two most efficient silencing sequences, shRap1A1 and shRap1A4, were selected and used to establish ESCC cell lines with stable knockdown of Rap1A (Fig. 2C).

In order to investigate the role of Rap1A in ESCC growth, the ESCC cell lines were used to evaluate cell proliferation. MTT analysis revealed that the downregulation of Rap1A suppressed the proliferation of KYSE150 cells compared with shRap1A-NC transfected cells (Fig. 2D). Consistently, colony formation assays revealed that the number of colonies formed by KYSE150-shRap1A1 and -shRap1A4 cells were significantly decreased compared with those formed by empty vector-transfected cells (Fig. 2E). However, the MTT and colony formation assays indicated that Rap1A did not affect ECA109 cell growth (Fig. 2D and E). In summary, the growth-promoting effect of Rap1A in ESCC is context-dependent.
RaplA knockdown decreases the migratory and invasive capacities of ESCC cells. To investigate the role of Rap1A in cell migration and invasion, a Transwell assay was performed after silencing Rap1A expression using shRap1A to evaluate ESCC cell motility. Both cell migration and invasion assays demonstrated that Rap1A knockdown significantly inhibited ECA109 cell migration and invasion (Fig. 3A and B). The same phenomenon was also observed in KYSE150-shRap1A1 and KYSE150-shRap1A4 cells compared with shRap1A-NC cells (Fig. 3C and D). These in vitro results suggest that Rap1A may promote the metastatic behavior of ESCC cells.

RaplA may enhance EMT via AKT signaling in ESCC cells. To identify the mechanism of action of Rap1A in ESCC, the changes in AKT-, pAKTser473- and EMT-associated signaling molecules in both ESCC cell lines were investigated following Rap1A knockdown. $\beta 1$ integrin has been reported to be stabilized by Rap1A and enhances migration of epithelial cells; therefore, it was included in the experiments (22). As shown in Fig. 4, siRNA-mediated downregulation of Rap1A resulted in the reduction of pAKT ser473 expression; furthermore, the expression of $\beta 1$ integrin, EMT markers and MMP9 were reduced compared with sh-NC. However, 
A

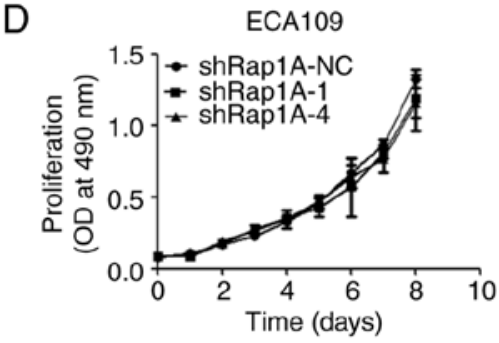

$\mathrm{E}$

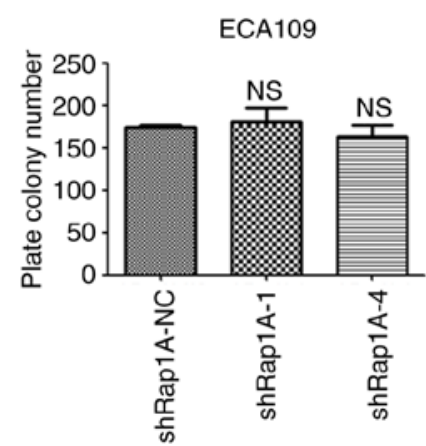

B
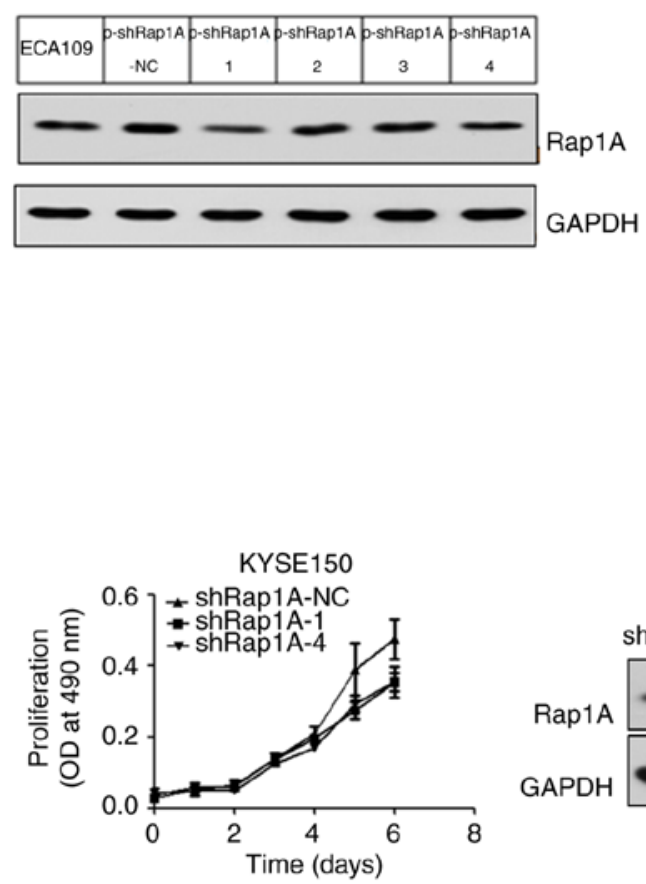
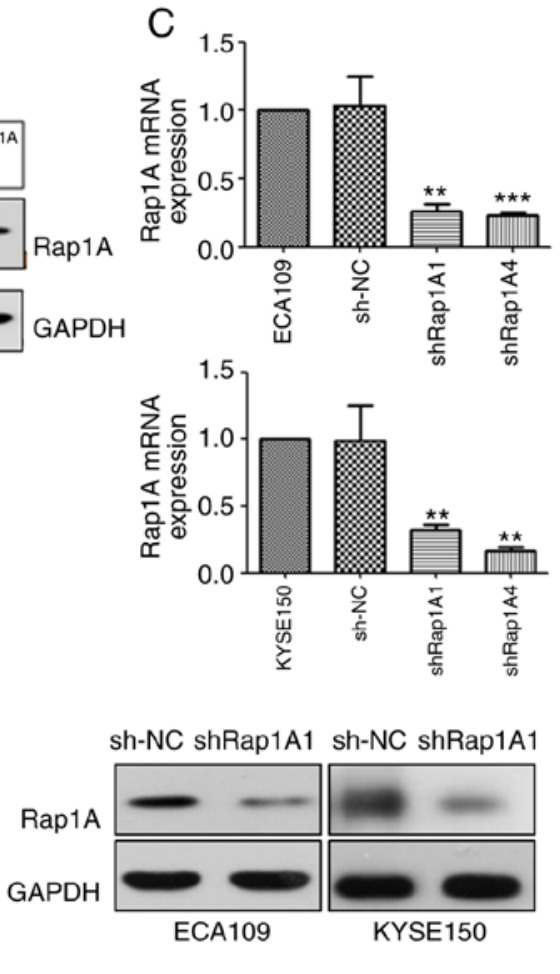

Figure 2. The growth-promoting effect of Rap1A in ESCC is context-dependent. (A and B) The Rap1A mRNA or protein expression level in ECA109 cells transfected with p-shRap1A1, p-shRap1A2, p-shRap1A3, p-shRap1A4 and the p-control. (C) RT-qPCR and western blot analysis results of Rap1A knockdown efficiency following transfection with lentiviral vectors containing one of the two most efficient shRap1A or LV-NC in ECA109 (upper panel) and KYSE150 (lower panel) cell lines. (D) Cell viability, determined by an MTT assay, as described in the methods. (E) The colony-forming ability of the indicated cells was measured using a colony formation assay after incubation for 15 days; error bars represent the mean \pm standard error of the mean; ${ }^{* *} \mathrm{P}<0.01{ }^{, * * *} \mathrm{P}<0.001$. Rap1A, Ras-associated protein 1A; ESCC, esophageal squamous cell carcinoma; RT-qPCR, reverse transcription-quantitative polymerase chain reaction.

the reduction of pAKT expression following Rap1A inhibition appeared to be minimal. The difference in AKT phosphorylation between the two cell lines was statistically significant $(\mathrm{P}<0.05)$. The statistical analysis of the AKT phosphorylation level following Rap1A inhibition was as follows: sh-NC vs. shRap1A, $1.000 \pm 0.015$ vs. $0.748 \pm 0.064$ in KYSE150 cells and $1.000 \pm 0.043$ vs. $0.774 \pm 0.041$ in ECA109 cells, respectively; unpaired t-test was applied to estimate the two-tailed P-values, which were 0.0188 and 0.019 , respectively. Therefore, the difference was statistically significant, although it was minimal after Rap1A inhibition. Thus, these results indicate that Rap1A may promote cancer cell migration and invasion via enhancement of $\beta 1$ integrin-dependent adhesion, and EMT and MMP9-associated degradation of the extracellular matrix; AKT signaling may participate in these processes.
SP1 upregulates human RaplA promoter activity through SP1-binding sites. The transcription factor SP1 plays an important role in ESCC (23). Consistently, we observed that SP1 could upregulate Rap1A expression in KYSE150 cells (Fig. 5A). To explore the molecular mechanism underlying Rap1A regulation by SP1, the CONSITE program was used to predict the SP1-specific binding sites in the Rap1A promoter (Fig. 5B), and then luciferase reporter and ChIP assays were performed in both ECA109 and KYSE150 cells. Overexpressing SP1 increased the luciferase activities of Rap1A promoter, Rap1A promoter mut1 and Rap1A promoter mut2 $(\mathrm{P}<0.001)$. The Rap1A promoter exhibited the highest luciferase activity, and SP1 overexpression in KYSE150 cells led to stronger Rap1A transactivation compared with that in ECA109 cells (Fig. 5C). The ChIP assay indicated that SP1 was capable of binding to the F1 


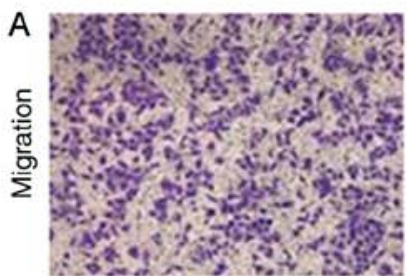

ECA109-shRap1A-NC
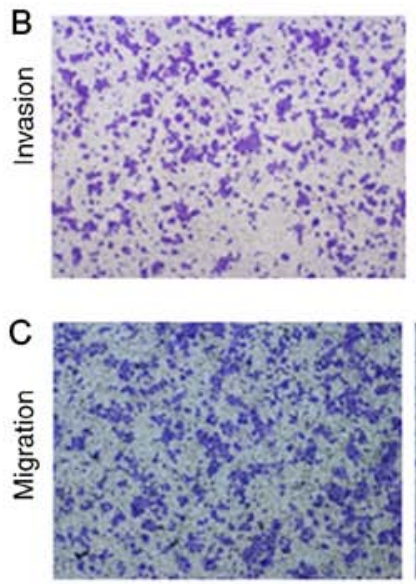

KYSE150-shRap1A-NC

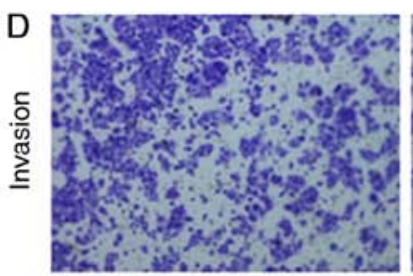

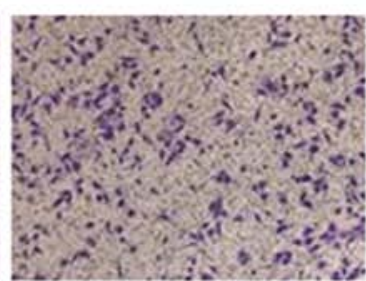

ECA109-shRap1A1
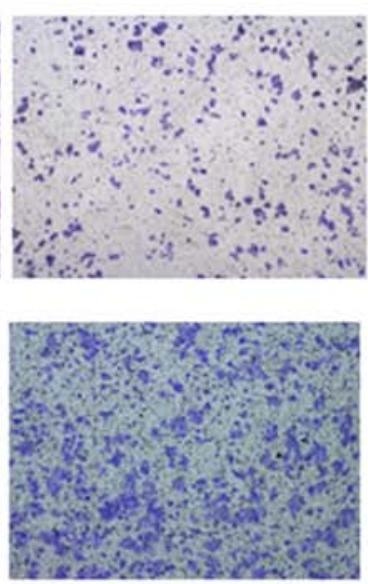

KYSE150-shRap1A-1

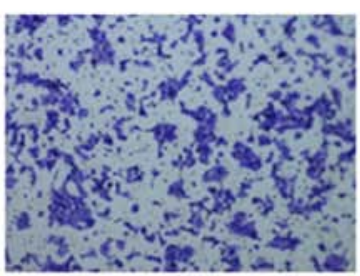

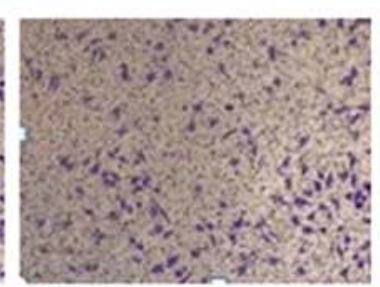

ECA109-shRap1A4
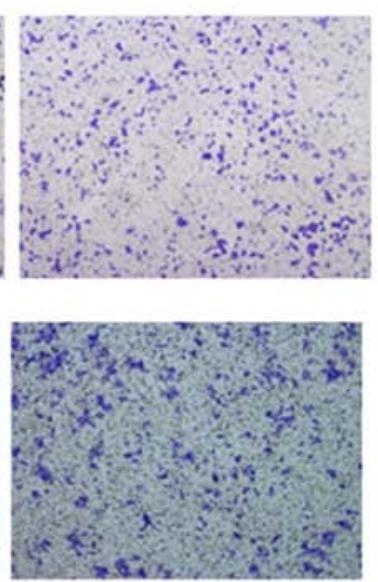

KYSE150-shRap1A-4

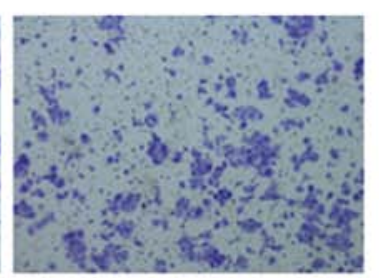

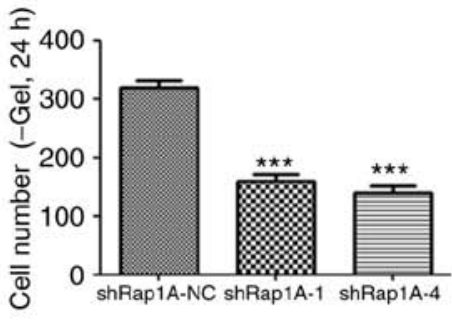
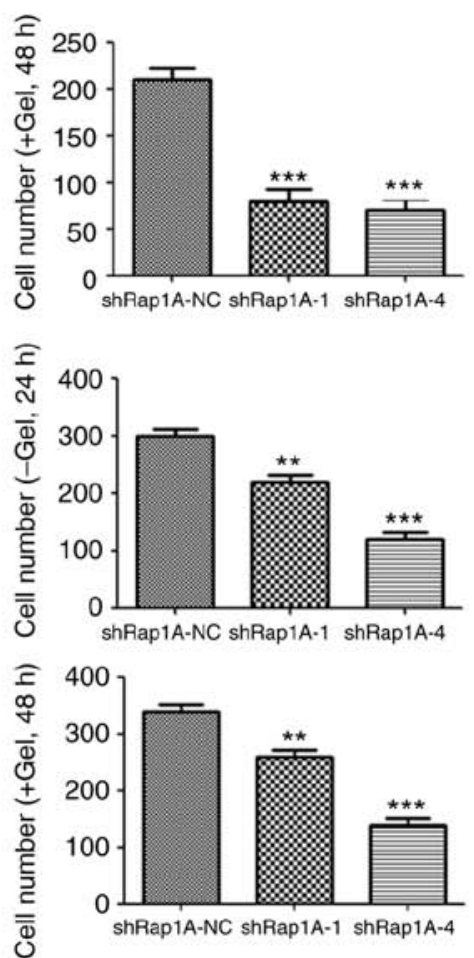

Figure 3. Rap1A knockdown decreases the migratory and invasive capacity of ESCC cells. (A) A Transwell assay was conducted to assess the effects of Rap1A knockdown on the migration and invasion abilities of ECA109 cells. (B) The number of invading ECA109 cells was quantified by counting the total number of cells. (C) A Transwell assay was conducted to assess the effects of Rap1A knockdown on the migration and invasion abilities of KYSE150 cells. (D) The number of invading KYSE150 cells was quantified by counting the total numbers of cells. Error bars represent the mean \pm standard error of the mean; ${ }^{* *} \mathrm{P}<0.01$,

${ }^{* * *} \mathrm{P}<0.001$. Rap1A, Ras-associated protein 1A; ESCC, esophageal squamous cell carcinoma.

(nt $-1,863$ to $-1,854$ ) and $\mathrm{F} 2$ (nt -882 to -873 ) regions within the Rap1A promoter. In addition, the ability of SP1 to bind to the F1 in KYSE150 cells was more prominent compared with that in ECA109 cells (Fig. 5D). These results suggest that SP1 upregulates human Rap1A promoter activity through two SP1-binding sites.

RaplA knockdown attenuates tumor metastasis in vivo. To determine the effect of Rap1A on ESCC tumorigenesis and metastasis in vivo, subcutaneous tumor and lung metastasis assays were performed in nude mice with ECA109 cells expressing shRap1A. For xenografted tumors, the results were valid and reliable, as all mice developed a single subcutaneous tumor (longest diameter, $16.53 \mathrm{~mm}$ ). The results indicated that ECA109 cells with Rap1A knockdown was associated with no significant difference in tumor size compared with control cells (Fig. 6A and B). In the metastasis model (Fig. 6C-F), the results of IHC and $\mathrm{H} \& \mathrm{E}$ staining indicated that downregulation of Rap1A decreased the number of metastatic nodules in the lungs. Taken together, these findings were consistent with the in vitro results and indicated that Rap1A promotes ESCC metastasis in vivo.

\section{Discussion}

The findings of the present study demonstrated the oncogenic function of Rap1A in the progression of ESCC. Rap1A promoted cell migration, invasiveness and metastasis, and its growth-promoting effect was found to be context-dependent. Moreover, Rap1A mediated EMT via AKT signaling and was regulated by the transcription factor SP1. Thus, Rap1A may be a potential therapeutic target for ESCC.

One of the novel findings of the present study was the activation of AKT signaling by the Rap1A small GTPase. It is well known that the Ras/Raf/MAPK pathway regulates a variety of cellular functions related to tumorigenesis (24). As the closest relative of Ras, Rap1A was previously shown to regulate MAPK/ERK signaling in solid tumors $(17,25,26)$. In addition to ERK, Rap1A promotes cancer cell proliferation via AKT signaling (27), and AKT activation plays a crucial role in the progression of ESCC, including cancer cell growth, migration, invasion and metastasis (11,28-30). With both AKT2 and KRAS amplification, enhanced alterations involving $\mathrm{PI} 3 \mathrm{~K} / \mathrm{AKT} / \mathrm{mTOR}$ signaling have been detected in the genomic profiling of ESCC $(31,32)$. Approximately $90 \%$ of tumors 
A

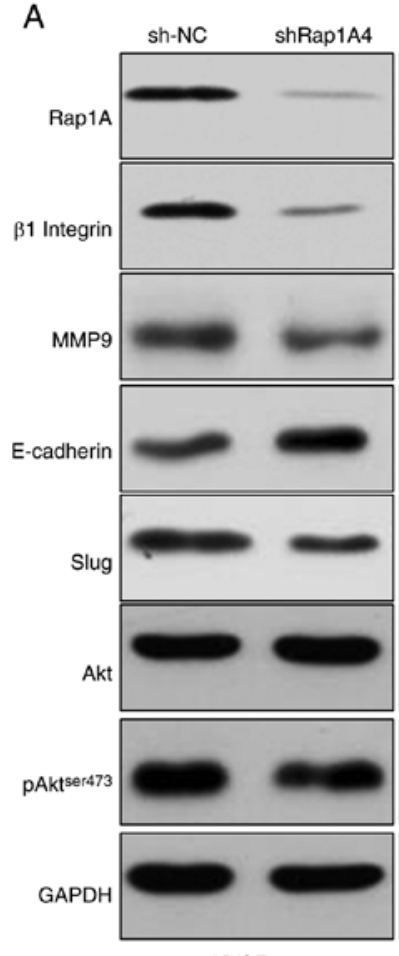

KYSE150

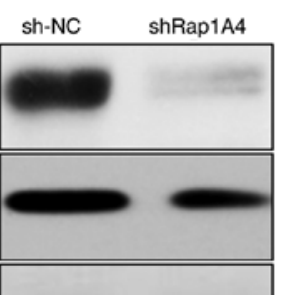

B
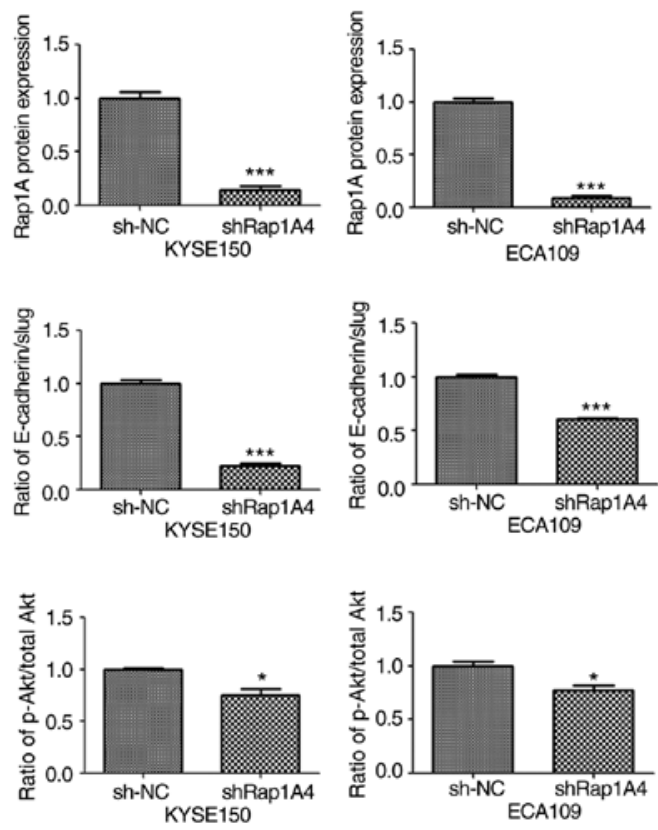

Figure 4. Rap1A enhances EMT via AKT signaling in ESCC cells. (A) Western blot analysis of the expression levels of AKT, $\beta 1$ integrin, MMP9, and epithelial ( $\beta$-catenin) and mesenchymal (Slug) markers in both ESCC cell lines following Rap1A knockdown. (B) The Rap1A knockdown efficiency and the degree of EMT and AKT phosphorylation were measured. Error bars represent the mean \pm standard error of the mean; ${ }^{*} \mathrm{P}<0.05,{ }^{* * * *} \mathrm{P}<0.001$. Rap1A, Ras-associated protein 1A; ESCC, esophageal squamous cell carcinoma; EMT, epithelial-to-mesenchymal transition; MMP, matrix metalloproteinase.

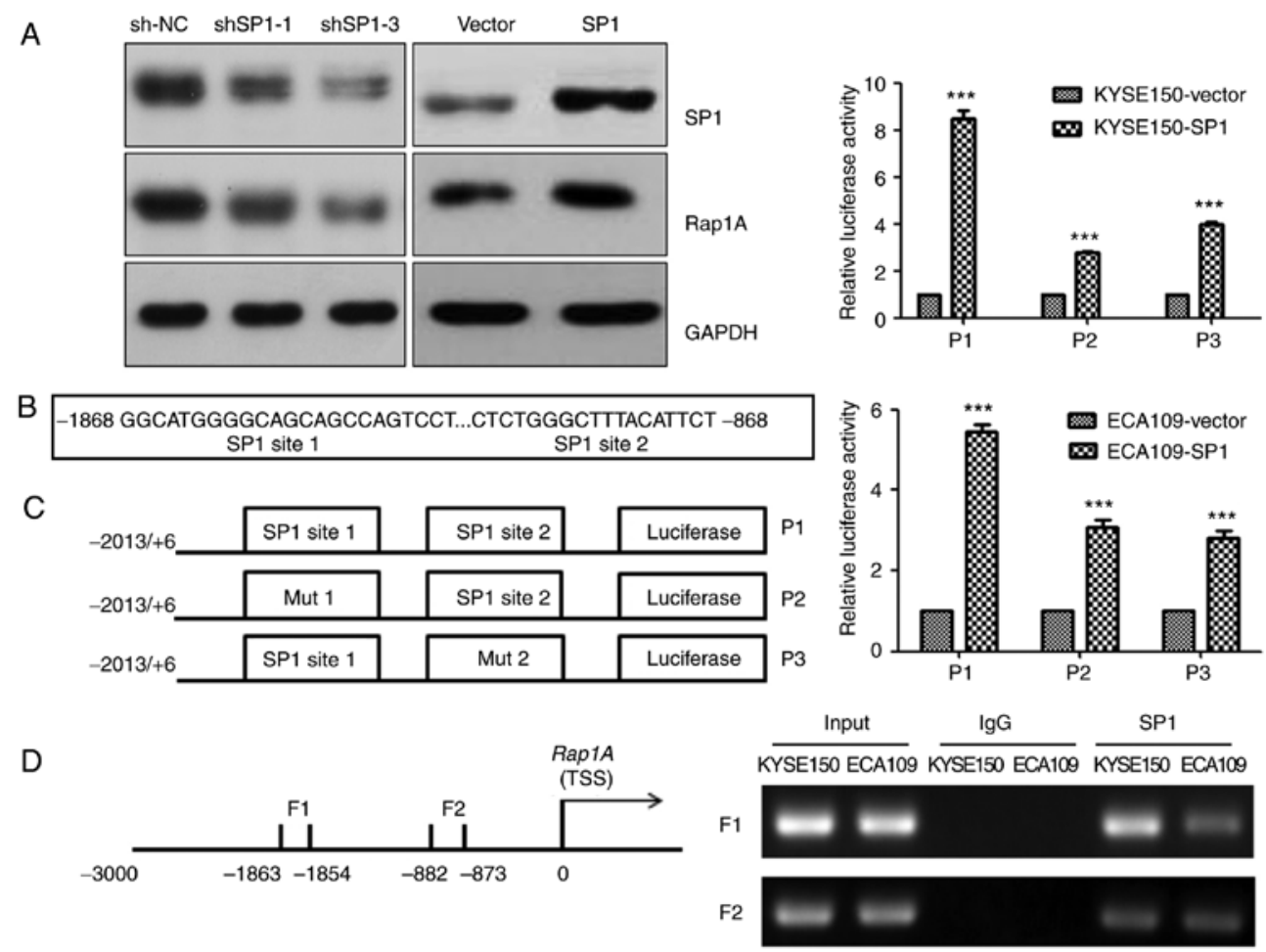

Figure 5. SP1 upregulates human Rap1A promoter activity through SP1-binding sites. (A) Western blot analysis of SP1 and Rap1A expression following the overexpression or downregulation of SP1 in KYSE150 cells. (B) Predictive sequence of the SP1-specific binding sites in the Rap1A promoter (shown in bold print). (C) Left panel: The Rap1A promoter and its two sites for directed mutagenesis were inserted upstream of fthe irefly luciferase coding sequences in the pGL3-basic plasmid. Right panel: Transactivation of the indicated serial Rap1A promoter fragments by SP1 in KYSE150 (upper panel) and ECA109 (lower panel) cells. Luciferase activity was normalized to Renilla luciferase activity; (D) Left panel: Schematic representation of PCR-amplified fragments of the Rap1A promoter. Right panel: ChIP assay using an SP1 antibody to identify SP1-binding sites in the Rap1A promoter. IgG was used as a negative control and Input was used as a positive control; error bars represent the mean \pm standard error of the mean; ${ }^{* * * *} \mathrm{P}<0.001$. Rap1A, Ras-associated protein $1 \mathrm{~A}$; ChIP, chromatin immunoprecipitation; PCR, polymerase chain reaction. 

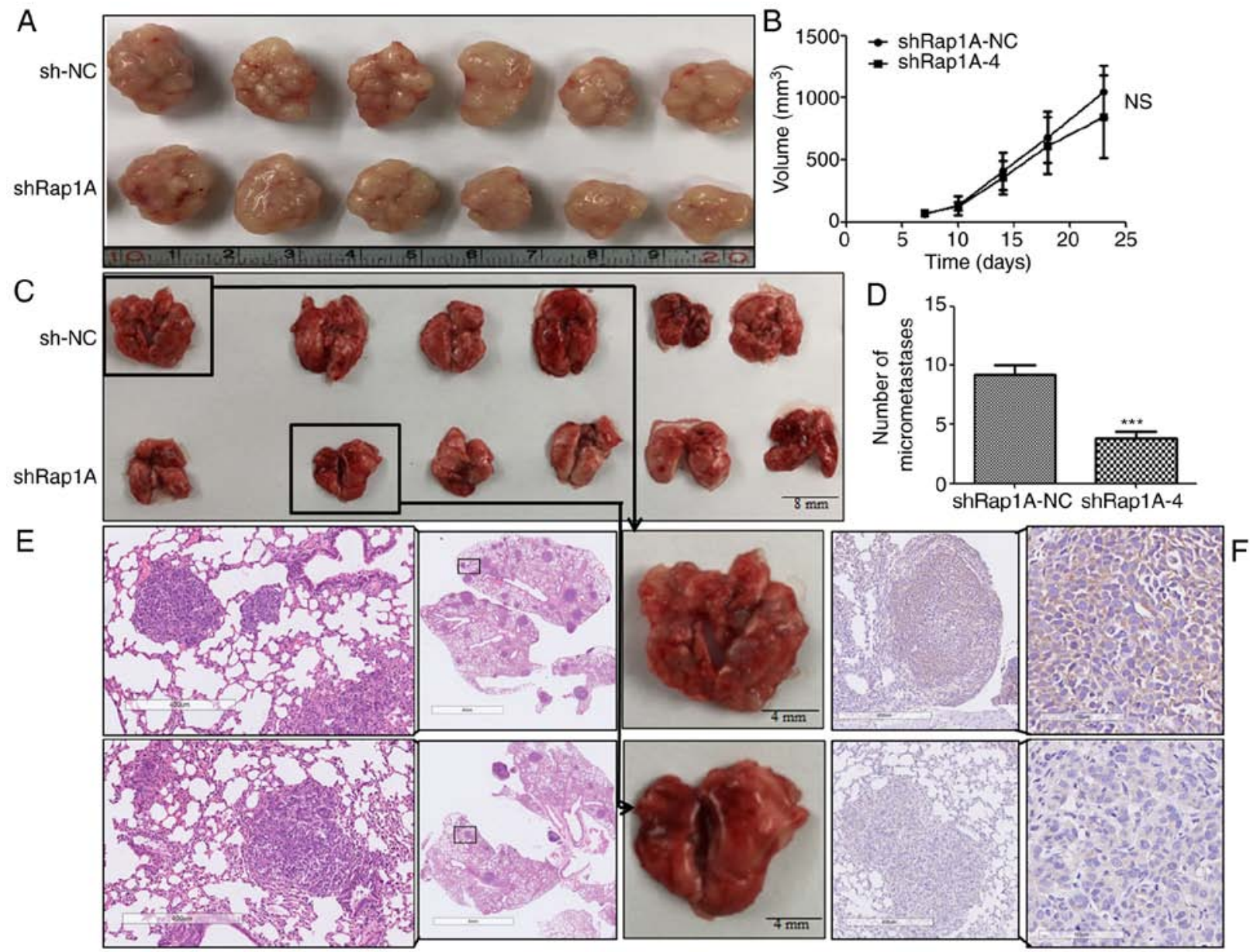

Figure 6. Rap1A knockdown attenuates tumor metastasis in vivo. (A) Images of ECA109 tumors using cells that stably expressed sh-NC or shRap1 A. (B) Quantitative analysis of the tumor volume with the time lapse from day 7 onwards; error bars represent mean $+95 \%$ confidence intervals, $\mathrm{n}=6$ mice/group (C) An illustrative diagram of lungs extracted 6 weeks after ECA109-sh-NC or shRap1A were injected into the tail veins of nude mice. (D) Quantitative analysis of the metastatic nodules in the lungs; error bars represent the mean \pm standard error of the mean; ${ }^{* * *} \mathrm{P}<0.001$. (E and F) Representative lung sections from each group and corresponding H\&E staining [(E) magnification, $\mathrm{x} 5$ and $\mathrm{x} 50]$ and $\mathrm{IHC}$ staining [(F) magnification, $\mathrm{x} 50$ and $\mathrm{x} 200]$ for Rap1A. Rap1A, Ras-associated protein $1 \mathrm{~A} ; \mathrm{H} \& \mathrm{E}$, hematoxylin and eosin; IHC, immunohistochemistry.

exhibit some degree of EMT during tumor development (11); notably, it was herein demonstrated that Rap1A activates AKT signaling to mediate EMT, and is regulated by the transcription factor SP1, which is consistent with the hypothesis that SP1 plays a key role in ESCC (23). Taken together, the findings of the present study combined with those of previous reports further indicated that AKT signaling may be positively regulated by Rap1A in ESCC.

Another important finding of the present research is that the oncogenic role of Rap1A in ESCC is context-dependent. It was previously reported that the function of miR-196a in ESCC is correlated with the SNPrs6573 A/C genotype in the Rap1A 3'-UTR (33). Furthermore, ECA109 and KYSE150 cells were found to be $\mathrm{CC}$ homozygotes, which interferes with the binding of miR-196a to Rap1A 3'-UTR, resulting in a higher constitutive expression of Rap1 A (33). In the present research, silencing Rap1A reversed the malignant phenotype of both cell lines, with the exception of ECA109 cell proliferation. It is important to note that both cell lines were isolated from the resected specimens of female patients with poorly differentiated ESCC $(34,35)$. Thus, the impact of Rap1A on ESCC cell growth is context-dependent and promotes tumor metastasis, which further supported previous reports.
Moreover, the reason why Rap1A is highly expressed in esophageal cancer was further investigated. In view of the important role of SP1 (23), it was hypothesized that the overexpression of Rap1A may be the result of the transcriptional activation of SP1.

In summary, the present study demonstrated that Rap1A promoted tumor metastasis, possibly via AKT signaling, integrin-mediated adhesion (27,36), MMP9 and EMT; furthermore, the growth-promoting effects of Rap1A in ESCC were associated with tumor heterogeneity, which required further research to elucidate the underlying mechanism. Considering the known amplification of the K-Ras gene in human esophageal cancer $(31,32,37)$, the expression of Rap1A may be an ESCC marker, and inhibitors of Rap1A may be of therapeutic value. In addition, mTOR plays a key role in regulating several fundamental cellular processes, and its hyperactivation is correlated with a number of human cancers (38). ESCC without lymph node metastasis may be effectively removed by endoscopic resection and can have a good prognosis $(39,40)$. Thus, further study investigating the specific mechanism involved in lymphatic metastasis is crucial for the treatment of ESCC.

There were certain limitations to the present study. It was demonstrated that Rap1 A was upregulated in human ESCC 
tissues compared with ANTT, but there is lack of data on human normal esophageal cells used as a negative control. The results showing that Rap1A promoted ESCC cell metastasis and that its growth-promoting effect was context-dependent relied on only two cell lines; thus, experiments on more cell lines are warranted to verify these findings. Furthermore, observation of the morphological changes was not performed, indicating a lack of effective verification regarding the presence of EMT. MMP2 is also a typical enzyme that degrades extracellular matrix. Thus, the absence of data on MMP2 is another limitation of the present study. It is also recommended to further investigate the roles of AKT and its associated mTOR pathway in ESCC. Despite these limitations, however, combining previous results (33) with the present research confirmed Rap1A as an oncogenic molecule in the progression of ESCC.

\section{Acknowledgements}

The authors would like to thank Dr Jia Cao (Department of Digestive Endoscopy, Shanghai East Hospital, Tongji University, China), for providing valuable suggestions on revising the manuscript.

\section{Funding}

The present study was supported by the National Natural Science Foundation of China (grant no. 81570595).

\section{Availability of data and materials}

The datasets used and/or analyzed during the present study are available from the corresponding author on reasonable request.

\section{Authors' contributions}

The study was conceived and designed by QL, AX, TC and MX. Study and clinical materials were provided by MX, PZ and LY. The experiments were performed by QL, AX, YC and HL. The datasets were analyzed by QL, AX and YC. The manuscript was written by QL, AX and TC. All authors have read and approved the final version of this manuscript for publication.

\section{Ethics approval and consent to participate}

Written informed consent was obtained from all patients prior to sample collection. The animal experiment were approved by Medical Ethics Committee of Fudan University (201906004Z) and all experiments on nude mice were performed according to the biosafety and bioethics guidelines.

\section{Patient consent for publication}

Not applicable.

\section{Competing interests}

All authors declare that they have no competing interests.

\section{References}

1. Chen W, Zheng R, Baade PD, Zhang S, Zeng H, Bray F, Jemal A, $\mathrm{Yu}$ XQ and He J: Cancer statistics in China, 2015. CA Cancer J Clin 66: 115-132, 2016.

2. Kuwano H, Nishimura Y, Oyama T, Kato H, Kitagawa $Y$, Kusano M, Shimada H, Takiuchi H, Toh Y, Doki Y, et al: Guidelines for diagnosis and treatment of carcinoma of the esophagus April 2012 edited by the Japan esophageal society. Esophagus 12: 1-30, 2015.

3. Pennathur A, Gibson MK, Jobe BA and Luketich JD: Oesophageal carcinoma. Lancet 381: 400-412, 2013.

4. Lagergren J, Smyth E, Cunningham D and Lagergren P: Oesophageal cancer. Lancet 390: 2383-2396, 2017.

5. Nitulescu GM, Van De Venter M, Nitulescu G, Ungurianu A, Juzenas P, Peng Q, Olaru OT, Grădinaru D, Tsatsakis A, Tsoukalas D, et al: The AKT pathway in oncology therapy and beyond (Review). Int J Oncol 53: 2319-2331, 2018.

6. Guanen Q, Junjie S, Baolin W, Chaoyang W, Yajuan Y, Jing L, Junpeng L, Gaili N, Zhongping W and Jun W: MiR-214 promotes cell metastasis and inhibits apoptosis of esophageal squamous cell carcinoma via PI3K/AKT/mTOR signaling pathway. Biomed Pharmacother 105: 350-361, 2018.

7. Zhao H, Chen G, Ye L, Yu H, Li S and Jiang WG: DOK7V1 influences the malignant phenotype of lung cancer cells through PI3K/AKT/mTOR and FAK/paxillin signaling pathways. Int J Oncol 54: 381-389, 2019.

8. Zhao J, Li Z, Chen Y, Zhang S, Guo L, Gao B, Jiang Y, Tian W, Hao $S$ and Zhang X: MicroRNA-766 inhibits papillary thyroid cancer progression by directly targeting insulin receptor substrate 2 and regulating the PI3K/AKT pathway. Int J Oncol 54: 315-325, 2019.

9. Liu SQ, Xu CY, Wu WH, Fu ZH, He SW, Qin MB and Huang JA: Sphingosine kinase 1 promotes the metastasis of colorectal cancer by inducing the epithelial-mesenchymal transition mediated by the FAK/AKT/MMPs axis. Int J Oncol 54: 41-52, 2019.

10. Dornan GL and Burke JE: Molecular mechanisms of human disease mediated by oncogenic and primary immunodeficiency mutations in Class IA phosphoinositide 3-kinases. Front Immunol 9: 575, 2018.

11. Jia Y, Xiao Z, Gongsun X, Xin Z, Shang B, Chen G, Wang Z and Jiang W: CEP55 promotes the proliferation, migration and invasion of esophageal squamous cell carcinoma via the PI3K/AKT pathway. Onco Targets Ther 11: 4221-4232, 2018.

12. Caron E: Cellular functions of the Rap1 GTP-binding protein: A pattern emerges. J Cell Sci 116: 435-440, 2003.

13. Khanna A, Lotfi P, Chavan AJ, Montaño NM, Bolourani P, Weeks G, Shen Z, Briggs SP, Pots H, Van Haastert PJ, et al: The small GTPases Ras and Rap1 bind to and control TORC2 activity. Sci Rep 6: 25823, 2016.

14. Sayyah J, Bartakova A, Nogal N, Quilliam LA, Stupack DG and Brown JH: The Ras-related protein, Rap1A, mediates thrombinstimulated, integrin-dependent glioblastoma cell proliferation and tumor growth. J Biol Chem 289: 17689-17698, 2014.

15. Grolez GP and Gkika D: TRPM8 puts the chill on prostate cancer. Pharmaceuticals (Basel) 9: pii: E44, 2016.

16. Genova T, Grolez GP, Camillo C, Bernardini M, Bokhobza A, Richard E,Scianna M,Lemonnier L, Valdembri D, Munaron L, et al: TRPM8 inhibits endothelial cell migration via a non-channel function by trapping the small GTPase Rap1. J Cell Biol 216: 2107-2130, 2017.

17. Lu L, Wang J, Wu Y, Wan P and Yang G: Rap1A promotes ovarian cancer metastasis via activation of ERK/p38 and notch signaling. Cancer Med 5: 3544-3554, 2016.

18. Rice TW, Ishwaran H, Ferguson MK, Blackstone EH and Goldstraw P: Cancer of the esophagus and esophagogastric junction: An eighth edition staging primer. J Thorac Oncol 12: 36-42, 2017.

19. Xiang J, Bian C, Wang H, Huang S and Wu D: MiR-203 down-regulates Rap1A and suppresses cell proliferation, adhesion and invasion in prostate cancer. J Exp Clin Cancer Res 34: 8, 2015.

20. Liu L, Yan X, Wu D, Yang Y, Li M, Su Y, Yang W, Shan Z, Gao Y and Jin Z: High expression of Ras-related protein 1 A promotes an aggressive phenotype in colorectal cancer via PTEN/FOXO3/CCND1 pathway. J Exp Clin Cancer Res 37: 178, 2018.

21. Chen $\mathrm{CH}$, Chuang HC, Huang CC, Fang FM, Huang HY, Tsai HT, Su LJ, Shiu LY, Leu S and Chien CY: Overexpression of Rap-1A indicates a poor prognosis for oral cavity squamous cell carcinoma and promotes tumor cell invasion via Aurora-A modulation. Am J Pathol 182: 516-528, 2013. 
22. To JY and Smrcka AV: Activated heterotrimeric G protein $\alpha$ subunits inhibit Rap-dependent cell adhesion and promote cell migration. J Biol Chem 293: 1570-1578, 2018.

23. Xiang J, Zang W, Che J, Chen K and Hang J: Regulation network analysis in the esophageal squamous cell carcinoma. Eur Rev Med Pharmacol Sci 16: 2051-2056, 2012.

24. Martinelli E, Morgillo F, Troiani T and Ciardiello F: Cancer resistance to therapies against the EGFR-RAS-RAF pathway: The role of MEK. Cancer Treat Rev 53: 61-69, 2017.

25. Zhang T, Jiang K, Zhu X, Zhao G, Wu H, Deng G and Qiu C: MiR-433 inhibits breast cancer cell growth via the MAPK signaling pathway by targeting Rap1A. Int J Biol Sci 14: 622-632, 2018.

26. Rodríguez CI, Castro-Pérez E, Prabhakar K, Block L, Longley BJ, Wisinski JA, Kimple ME and Setaluri V: EPAC-RAP1 axis-mediated switch in the response of primary and metastatic melanoma to cyclic AMP. Mol Cancer Res 15: 1792-1802, 2017.

27. Park JY and Juhnn YS: cAMP signaling increases histone deacetylase 8 expression via the Epac2-Rap1A-AKT pathway in H1299 lung cancer cells, histone deacetylases and cancer. Exp Mol Med 49: e297, 2017.

28. Liu M, Hu Y, Zhang MF, Luo KJ, Xie XY, Wen J, Fu JH and Yang H: MMP1 promotes tumor growth and metastasis in esophageal squamous cell carcinoma. Cancer Lett 377: 97-104, 2016.

29. Zhang J, Kong R and Sun L: Silencing of Rab3D suppresses the proliferation and invasion of esophageal squamous cell carcinoma cells. Biomed Pharmacother 91: 402-407, 2017.

30. Kong KL, Kwong DL, Chan TH, Law SY, Chen L, Li Y, Qin YR and Guan XY: MicroRNA-375 inhibits tumour growth and metastasis in oesophageal squamous cell carcinoma through repressing insulin-like growth factor 1 receptor. Gut 61: 33-42, 2012.

31. Yang JW and Choi YL: Genomic profiling of esophageal squamous cell carcinoma (ESCC)-Basis for precision medicine. Pathol Res Pract 213: 836-841, 2017.
32. Wang K, Johnson A, Ali SM, Klempner SJ, Bekaii-Saab T, Vacirca JL, Khaira D, Yelensky R, Chmielecki J, Elvin JA, et al: Comprehensive genomic profiling of advanced esophageal squamous cell carcinomas and esophageal adenocarcinomas reveals similarities and differences. Oncologist 20: 1132-1139, 2015.

33. Wang K, Li J, Guo H, Xu X, Xiong G, Guan X, Liu B, Li J, Chen X, Yang K, et al: MiR-196a binding-site SNP regulates Rap1A expression contributing to esophageal squamous cell carcinoma risk and metastasis. Carcinogenesis 33: 2147-2154, 2012.

34. Shimada Y, Imamura M, Wagata T, Yamaguchi $\mathrm{N}$ and Tobe $\mathrm{T}$. Characterization of 21 newly established esophageal cancer cell lines. Cancer 69: 277-284, 1992.

35. Xiao F, Wang X, Wang M, Guan X and Wu M: Molecular cytogenetic study on four human esophageal cancer cell lines. Zhonghua Yi Xue Yi Chuan Xue Za Zhi 15: 75-77, 1998 (In Chinese).

36. Tsygankova OM, Ma C, Tang W, Korch C, Feldman MD, Lv Y, Brose MS and Meinkoth JL: Downregulation of Rap1GAP in human tumor cells alters cell matrix and cell cell adhesion. Mol Cell Biol 30: 3262-3274, 2010.

37. Salem ME, Puccini A, Xiu J, Raghavan D, Lenz HJ, Korn WM, Shields AF, Philip PA, Marshall JL and Goldberg RM: Comparative molecular analyses of esophageal squamous cell carcinoma, esophageal adenocarcinoma, and gastric adenocarcinoma. Oncologist 23: 1319-1327, 2018.

38. Saxton RA and Sabatini DM: mTOR signaling in growth, metabolism, and disease. Cell 168: 960-976, 2017.

39. Aadam AA and Abe S: Endoscopic submucosal dissection for superficial esophageal cancer. Dis Esophagus 31, 2018.

40. Zhang YQ, Chen T, Zhang C, Li QL, Chen WF, Yao LQ, Zhou PH and Xu MD: Endoscopic submucosal dissection for superficial proximal esophageal neoplasia is highly successful. Ann Surg 266: 995-999, 2017.

This work is licensed under a Creative Commons Attribution-NonCommercial-NoDerivatives 4.0 International (CC BY-NC-ND 4.0) License. 